\title{
Capitation funding: population, age, and mortality adjustments for regional and district health authorities in England
}

\author{
James Raftery
}

\begin{abstract}
Objective-To assess the effect of each of the components of the national capitation funding formula-population projections and age and mortality weighting - at regional and district level.

Design-Application of age-cost and mortality weights to the projected 1997 populations of regions and districts, based on the Department of Health public health common dataset.

Setting-Regional and district health authorities in England.

Results-The application of the age-cost and mortality weights to projected populations resulted in greater changes in the shares of weighted populations relative to the estimated 1991 population at district level (mean 0 , range $-17 \%$ to $28 \%$ ) than at regional level (mean 0 , range $-9 \%$ to $6 \%$ ). At district level mortality weights had less scope for influence (mean 0 , range $-9 \%$ to $14 \%$ ) than population projections (mean 0 , range $-16 \%$ to $31 \%$ ) or age weights (mean 0 , range $-20 \%$ to $30 \%)$.
\end{abstract}

Conclusions-The adjustments to the 1991 population shares due to the application of the national capitation funding formula depend on the interaction of three elements: the projected population by age group, the age-cost weight, and the mortality weight. Since each is open to uncertainty, either in terms of measurement (projected population, particularly for births and the over 85s) or method (derivation of the age-cost and mortality weights), the formula should be implemented cautiously at district level. Ways should be considered of incorporating elements of uncertainty into the model. Further research is required on the elements and degree of uncertainty in each of these components, as well as on the relative health needs of different populations.

\section{Introduction}

Capitation funding, whereby health authorities receive NHS funds on the basis of the relative size and composition of their resident populations, is one of the distinguishing characteristics of the reformed National Health Service, although it has existed for much longer. ${ }^{1}$ In 1976, for example, the Resource Allocation Working Party (RAWP) set funding targets for regions based on populations weighted for age, sex, and mortality with adjustments for cross boundary flows, local costs (such as London weighting), and teaching. ${ }^{2}$ Capitation funding implies a strong commitment to geographical equity by providing equal public funding for health care per head throughout England and Wales, with the age and mortality weightings reflecting judgments about differential needs.

Following RAWP, disparities in funding between the 14 regions narrowed from a $27 \%$ range between the best off and the least well off in $1977-8^{3}$ to $4 \%$ in 1986-7. ${ }^{1}$ The NHS reforms of 1991 led to regions being funded entirely on the basis of weighted capitation in 1992-3. Before 1991 regions applied various forms of subregional capitation to districts, based around the share of hospital centred "planning populations" in the region's total population. By separating purchasers and providers and funding the purchaser for resident as opposed to planning populations the NHS reforms made weighted capitation more important at district level. ${ }^{4}$ In applying capitation funding to districts, however, regions are permitted to make adjustmentsfor example, over the pace of change and for social deprivation. Since different social deprivation indexes give different results, many regions are currently engaged in lively debates about which to use.

The main focus hitherto in the debate on capitation funding has been on the appropriate adjustments for mortality (taken to be a proxy for morbidity) and for social deprivation. ${ }^{5}$ Less attention has been paid to the impact of population projections and age weights. This paper assesses the contribution of each of the three main elements in the national formula-population projections and age and mortality weights-to the changes implied by capitation targets.

\section{Elements in the formula}

The age-cost weights used in the national formula for hospital and community health services (table I) indicate relatively high expenditure at birth and on each of the elderly age groups, especially the over $85 \mathrm{~s}$, whose average spend is over $£ 2000$ a year. Sexes are not weighted separately. Since the average overall spending on these services was $£ 308$ a head per year, births and the over $85 \mathrm{~s}$ cost five to seven times that average. The weights ${ }^{6}$ are based on the national average age-use profiles of 29 programmes $^{7}$ and apply to the whole hospital and community health services budget. The 1993-4 age-cost weights, used in this analysis, average the three years $1988-91$.

Mortality adjustment-The RAWP formula adjusted populations for morbidity by using disease specific standardised mortality ratios to give "need weighted populations." In 1987, on the basis of multivariate analysis of differences in hospitalisation rates, a national review suggested that the mortality adjustment should be based on the square root of the all cause standardised mortality ratio and that the relevant standardised mortality ratio should be that for the population aged under $75 .^{8}$ These suggestions were incorporated into the national formula for weighted capitation.

The national capitation formula takes some accoun of random variation by using five year standardised mortality ratios and three year average age weights. 
Since regions contain 2-5 million residents the level of random variation to be expected in applying age-cost or mortality weights is relatively small. The greater level of random variation in mortality to be expected in districts, which typically have populations of 250000 500000 , has generated considerable debate. ${ }^{9-12}$ The variations likely to be associated with particular age groups have received less attention. ${ }^{13}$

\section{Design and methods}

This study replicates the core elements of the national capitation formula by combining publicly available data on projected populations by age group with age and mortality weights at both regional and district levels. Although use of the publicly available data necessitates some slight departures from the national method, these are small enough to enable the broad effects of capitation weighting to be assessed. The national formula requires data on three elements: (a) mid-year population projections by age group for the year of allocation, ${ }^{\prime}(b)$ application of age-cost weights to that population by age group, and (c) adjustment of the total population by the square root of the all cause standardised mortality ratio for people aged under 75 .

The public health common dataset provides estimates of mid-year 1991 populations by age group with similar projections for $1997 .{ }^{14}$ Although these six year projections refer to a slightly longer period than the five year planning horizons of most regions, they indicate the likely scale of population changes.

The age-cost weights shown in table I were applied to each age group for 1997 and the resulting expenditure aggregated. The share of each health authority in total expenditure was used to drive an age weighted population, which, although the same at national level (mean change 0 ), shows differences at subnational levels.

The public health common dataset also provides data on several all cause standardised mortality ratios by regional and district health authority: that for all ages, for under $15 \mathrm{~s}$, for the 15-64 age group, and for the over $65 \mathrm{~s}$. Given the lack of a measure relating specifically to the population under 75 , the all age, all cause standardised mortality ratio was used. Mortality weights were derived by taking the square root of this ratio. As with the age weights, the mortality weighted population was identical with the projected 1997 population at national level (mean change 0 ) but not at subnational levels.

Since the share of national population in each region and district, rather than absolute population size, determines capitation targets the changes in shares due to the various elements were calculated relative to 1991 shares.

\section{Results}

OVERALL EFFECTS

Regional level-The application of these various weights to the projected 1997 populations resulted in considerable changes at regional level (table II), with a mean change of zero on the 1991 population and a range from $-9 \%$ (North West Thames region) to $6 \%$ (South Western region). Population projections accounted for slightly less of the projected changes (mean 0 , range -3 to $4 \%$ ) than age weights (mean 0 , range $-6 \%$ to $7 \%$ ) or mortality (mean 0 , range $-4 \%$ to $7 \%$ ). Since the changes implied by each component tended to offset each other in most regions, the range of their combined effects was only slightly greater than that for each component.

District level-Although the mean change nationally was 0 , much greater swings occurred at district level, with losses of up to $-17 \%$ and gains of up to $28 \%$. Table III shows the projected changes in share of national weighted population compared with 1991 for the top two overall gainer and loser districts in each region. A few districts gained by over $20 \%$, though none lost by more than $17 \%$ (Haringey and Hampstead). Overall 99 districts were losers and 87 gainers. All regions except one had some gainers and losers, but two (Northern and South Western) had only three losers. By contrast, all the districts in North West Thames and all but one in North East Thames were losers. The widest spread of losers and gainers was in South East Thames $(-15 \%$ to $28 \%$ ) and South West Thames $(-14 \%$ to $27 \%)$.

\section{POPULATION PROJECTION EFFECTS}

Population projections had a very different pattern from the total change, with many of the districts which were large gainers in total capitation projected to lose on the population projection element. The range of change in projected population share was from $-16 \%$ to $31 \%$ around a mean of zero. Largest projected increases were for the "new towns" of Milton Keynes and Huntingdon. The largest losers included older urban areas such as North Manchester, Central Manchester, Liverpool, Hull, Haringey, and Hampstead.

\section{AGE-COST WEIGHTS}

Table III shows the effects due to age-cost weights. The mean change was 0 , with a range of $-20 \%$ to $30 \%$. Some districts lost heavily due to age weighting, including towns such as Milton Keynes and Huntingdon which were projected to have large total population increases. Projected population increases in these districts were mainly in the younger age groups, which had relatively low age-cost weights.

All regions had gainers and losers, with a strong tendency for the gainers to be seaside resorts (because

TABLE II-Estimated and projected weighted populations, with percentage changes in population share for regional health authorities in England 1991 and $1997^{7} 15$

\begin{tabular}{|c|c|c|c|c|c|c|c|c|}
\hline \multirow[b]{2}{*}{ Region } & \multirow[b]{2}{*}{$\begin{array}{c}\text { Estimated } \\
\text { population } \\
1991\end{array}$} & \multirow[b]{2}{*}{$\begin{array}{c}\text { Projected } \\
\text { population } \\
1997\end{array}$} & \multirow[b]{2}{*}{$\begin{array}{c}\text { Age weighted } \\
\text { population } \\
1997\end{array}$} & \multirow{2}{*}{$\begin{array}{c}\text { Mortality } \\
\text { weighted } \\
\text { population } \\
1997\end{array}$} & \multicolumn{4}{|c|}{$\%$ Changes in population share due to: } \\
\hline & & & & & $\begin{array}{l}\text { Population } \\
\text { projection }\end{array}$ & $\begin{array}{c}\text { Age } \\
\text { weight }\end{array}$ & $\begin{array}{c}\text { Mortality } \\
\text { weight }\end{array}$ & $\begin{array}{l}\text { Combined } \\
\text { effects }\end{array}$ \\
\hline Northern & 3077265 & 3063568 & 3048405 & 3246613 & $-2 \cdot 39$ & -0.49 & 6.50 & 3.44 \\
\hline Yorkshire & 3673134 & 3722729 & 3728290 & 3811418 & -0.63 & $0 \cdot 15$ & $2 \cdot 23$ & 1.74 \\
\hline Trent & 4721798 & 4822283 & 4779754 & 4855779 & $0 \cdot 13$ & -0.88 & 1.59 & 0.83 \\
\hline East Anglian & 2083650 & 2189324 & 2264445 & 2177697 & 3.02 & $3 \cdot 43$ & $-3 \cdot 83$ & $2 \cdot 47$ \\
\hline North West Thames & 3566541 & 3593289 & 3436920 & 3296291 & $-1 \cdot 22$ & -4.35 & -4.09 & $-9 \cdot 38$ \\
\hline North East Thames & 3759039 & 3798278 & 3709451 & 3628658 & -0.93 & $-2 \cdot 34$ & $-2 \cdot 18$ & $-5 \cdot 36$ \\
\hline South East Thames & 3671311 & 3763819 & 3872067 & 3787732 & 0.51 & 2.88 & $-2 \cdot 18$ & $1 \cdot 15$ \\
\hline South West Thames & 2985216 & 3023141 & 3103752 & 2970263 & -0.71 & $2 \cdot 67$ & $-4 \cdot 30$ & $-2 \cdot 45$ \\
\hline Wessex & 2955108 & 3112419 & 3239322 & 3110160 & $3 \cdot 26$ & 4.08 & -3.99 & $3 \cdot 19$ \\
\hline Oxford & 2578359 & 2729734 & 2554979 & 2471699 & 3.80 & $-6 \cdot 40$ & $-3 \cdot 26$ & $-6 \cdot 01$ \\
\hline South Western & 3271643 & 3435961 & 3673093 & 3545750 & $2 \cdot 97$ & $6 \cdot 90$ & -3.47 & $6 \cdot 26$ \\
\hline West Midlands & 5228424 & 5300899 & 5211934 & 5343445 & -0.60 & $-1 \cdot 68$ & $2 \cdot 52$ & $0 \cdot 20$ \\
\hline Mersey & 2406555 & 2384109 & 2372925 & 2482162 & $-2 \cdot 87$ & -0.47 & $4 \cdot 60$ & $1 \cdot 12$ \\
\hline North Western & 4030378 & 4026436 & 3970651 & 4210104 & $-2 \cdot 05$ & $-1 \cdot 39$ & 6.03 & $2 \cdot 42$ \\
\hline England & 48008421 & 48965977 & 48965926 & 48965926 & 0.00 & 0.00 & 0.00 & 0.00 \\
\hline
\end{tabular}


of their high proportion of retired people) and the losers inner cities.

MORTALITY WEIGHTS

The effect of mortality weights is also shown in table III. The mean contribution of mortality weights was equal to 0 and ranged from $-9 \%$ to $14 \%$. The northern districts tended to gain owing to their raised relative mortality compared with the south. Against this trend, several of the inner London districts had raised mortality and hence gained due to this weighting.

TABLE III-Estimated and projected weighted populations, with percentage changes in population share for district health authorities in England 1991 and $1977^{715}$

\begin{tabular}{|c|c|c|c|c|c|c|c|c|}
\hline \multirow[b]{2}{*}{ District } & \multirow{2}{*}{$\begin{array}{c}\text { Estimated } \\
\text { population } \\
1991\end{array}$} & \multirow{2}{*}{$\begin{array}{c}\text { Projected } \\
\text { population } \\
1997\end{array}$} & \multirow{2}{*}{$\begin{array}{c}\text { Age weighted } \\
\text { population } \\
1997\end{array}$} & \multirow{2}{*}{$\begin{array}{c}\text { Mortality } \\
\text { weighted } \\
\text { population } \\
1997\end{array}$} & \multicolumn{4}{|c|}{$\%$ Changes in population share due to: } \\
\hline & & & & & $\begin{array}{l}\text { Population } \\
\text { projection }\end{array}$ & $\begin{array}{c}\text { Age } \\
\text { weight }\end{array}$ & $\begin{array}{l}\text { Mortality } \\
\text { weight }\end{array}$ & $\begin{array}{c}\text { Combined } \\
\text { effects }\end{array}$ \\
\hline \multicolumn{9}{|c|}{ Northern region } \\
\hline $\begin{array}{l}\text { Hartlepool } \\
\text { Durham } \\
\text { Darlington } \\
\text { South Cumbria }\end{array}$ & $\begin{array}{r}89927 \\
236577 \\
125191 \\
170723\end{array}$ & $\begin{array}{r}83950 \\
229340 \\
125987 \\
178395\end{array}$ & $\begin{array}{r}82371 \\
226068 \\
126260 \\
191192\end{array}$ & $\begin{array}{r}89492 \\
238309 \\
139312 \\
190135\end{array}$ & $\begin{array}{r}-8.47 \\
-4.95 \\
-1.33 \\
2.45\end{array}$ & $\begin{array}{r}-1 \cdot 88 \\
-1 \cdot 43 \\
0 \cdot 22 \\
7 \cdot 17\end{array}$ & $\begin{array}{r}8 \cdot 64 \\
5 \cdot 41 \\
10 \cdot 34 \\
-0.55\end{array}$ & $\begin{array}{r}-2 \cdot 43 \\
-1 \cdot 24 \\
9 \cdot 10 \\
9 \cdot 19\end{array}$ \\
\hline \multicolumn{9}{|c|}{ Yorkshire region } \\
\hline $\begin{array}{l}\text { Pontefract } \\
\text { Hull } \\
\text { Scarborough } \\
\text { East Yorkshire }\end{array}$ & $\begin{array}{l}171466 \\
298103 \\
150407 \\
206351\end{array}$ & $\begin{array}{l}167346 \\
271723 \\
153923 \\
238886\end{array}$ & $\begin{array}{l}160819 \\
284060 \\
175967 \\
241938\end{array}$ & $\begin{array}{l}169144 \\
296046 \\
173749 \\
238766\end{array}$ & $\begin{array}{r}-4 \cdot 31 \\
-10 \cdot 63 \\
0 \cdot 34 \\
13 \cdot 50\end{array}$ & $\begin{array}{r}-3 \cdot 90 \\
4 \cdot 54 \\
14 \cdot 32 \\
1 \cdot 28\end{array}$ & $\begin{array}{r}5 \cdot 18 \\
4 \cdot 22 \\
-1 \cdot 26 \\
-1 \cdot 31\end{array}$ & $\begin{array}{r}-3 \cdot 28 \\
-2 \cdot 63 \\
13 \cdot 26 \\
13 \cdot 45\end{array}$ \\
\hline \multicolumn{9}{|c|}{ Trent region } \\
\hline $\begin{array}{l}\text { Leicestershire } \\
\text { Nottingham } \\
\text { South Lincolnshire } \\
\text { North Lincolnshire }\end{array}$ & $\begin{array}{l}903789 \\
621042 \\
318356 \\
278010\end{array}$ & $\begin{array}{l}936135 \\
617727 \\
334611 \\
287794\end{array}$ & $\begin{array}{l}913175 \\
612252 \\
344689 \\
287319\end{array}$ & $\begin{array}{l}895621 \\
615922 \\
341392 \\
300408\end{array}$ & $\begin{array}{r}1.55 \\
-2.48 \\
3.05 \\
1.49\end{array}$ & $\begin{array}{r}-2.45 \\
-0.89 \\
3.01 \\
-0.16\end{array}$ & $\begin{array}{r}-1.92 \\
0.60 \\
-0.96 \\
4.56\end{array}$ & $\begin{array}{r}-2 \cdot 84 \\
-2 \cdot 76 \\
5 \cdot 14 \\
5 \cdot 94\end{array}$ \\
\hline \multicolumn{9}{|c|}{ East Anglian region } \\
\hline $\begin{array}{l}\text { Cambridge } \\
\text { Huntingdon } \\
\text { West Norfolk and Wisbech } \\
\text { Great Yarmouth and Waveney }\end{array}$ & $\begin{array}{l}284692 \\
142947 \\
193165 \\
203850\end{array}$ & $\begin{array}{l}279239 \\
172390 \\
208256 \\
216602\end{array}$ & $\begin{array}{l}280122 \\
147612 \\
220093 \\
236502\end{array}$ & $\begin{array}{l}262592 \\
142034 \\
214169 \\
229527\end{array}$ & $\begin{array}{r}-3 \cdot 83 \\
18 \cdot 24 \\
5 \cdot 70 \\
4 \cdot 18\end{array}$ & $\begin{array}{r}0 \cdot 32 \\
-14 \cdot 37 \\
5 \cdot 68 \\
9 \cdot 19\end{array}$ & $\begin{array}{l}-6 \cdot 26 \\
-3 \cdot 78 \\
-2 \cdot 69 \\
-2 \cdot 95\end{array}$ & $\begin{array}{r}-9 \cdot 57 \\
-2 \cdot 58 \\
8 \cdot 71 \\
10 \cdot 39\end{array}$ \\
\hline \multicolumn{9}{|c|}{ North West Thames region } \\
\hline $\begin{array}{l}\text { Ealing } \\
\text { Barnet } \\
\text { North Hertfordshire } \\
\text { North Bedfordshire }\end{array}$ & $\begin{array}{l}291911 \\
312944 \\
188588 \\
256011\end{array}$ & $\begin{array}{l}287057 \\
309658 \\
190496 \\
270788\end{array}$ & $\begin{array}{l}265598 \\
288469 \\
185664 \\
250908\end{array}$ & $\begin{array}{l}258039 \\
277418 \\
182285 \\
249268\end{array}$ & $\begin{array}{r}-3 \cdot 59 \\
-2 \cdot 99 \\
-0.96 \\
3 \cdot 70\end{array}$ & $\begin{array}{l}-7 \cdot 48 \\
-6 \cdot 84 \\
-2 \cdot 54 \\
-7 \cdot 34\end{array}$ & $\begin{array}{l}-2 \cdot 85 \\
-3 \cdot 83 \\
-1 \cdot 82 \\
-0.65\end{array}$ & $\begin{array}{r}-13.33 \\
-13.09 \\
-5 \cdot 23 \\
-4.54\end{array}$ \\
\hline \multicolumn{9}{|c|}{ North East Thames region } \\
\hline $\begin{array}{l}\text { Haringey } \\
\text { Hampstead } \\
\text { West Essex } \\
\text { North East Essex }\end{array}$ & $\begin{array}{l}194749 \\
112632 \\
249102 \\
321227\end{array}$ & $\begin{array}{l}180365 \\
103727 \\
257266 \\
348120\end{array}$ & $\begin{array}{l}164926 \\
101706 \\
259380 \\
372658\end{array}$ & $\begin{array}{r}165091 \\
95883 \\
253863 \\
355262\end{array}$ & $\begin{array}{r}-9 \cdot 20 \\
-9 \cdot 71 \\
1 \cdot 26 \\
6 \cdot 25\end{array}$ & $\begin{array}{r}-8.56 \\
-1.95 \\
0.82 \\
7.05\end{array}$ & $\begin{array}{r}0 \cdot 10 \\
-5 \cdot 73 \\
-2 \cdot 13 \\
-4 \cdot 67\end{array}$ & $\begin{array}{r}-16.89 \\
-16.54 \\
-0.08 \\
8.43\end{array}$ \\
\hline \multicolumn{9}{|c|}{ South East Thames region } \\
\hline $\begin{array}{l}\text { Bexley } \\
\text { Camberwell } \\
\text { Eastbourne } \\
\text { Hastings }\end{array}$ & $\begin{array}{l}221778 \\
211727 \\
246495 \\
168963\end{array}$ & $\begin{array}{l}215512 \\
204135 \\
269585 \\
182605\end{array}$ & $\begin{array}{l}204329 \\
188324 \\
332062 \\
228789\end{array}$ & $\begin{array}{l}192414 \\
190855 \\
304463 \\
220620\end{array}$ & $\begin{array}{r}-4 \cdot 73 \\
-5 \cdot 47 \\
7 \cdot 23 \\
5 \cdot 96\end{array}$ & $\begin{array}{l}-5 \cdot 19 \\
-7 \cdot 75 \\
23 \cdot 18 \\
25 \cdot 29\end{array}$ & $\begin{array}{r}-5 \cdot 83 \\
1.34 \\
-8 \cdot 31 \\
-3.57\end{array}$ & $\begin{array}{r}-14 \cdot 94 \\
-11 \cdot 62 \\
21 \cdot 10 \\
28 \cdot 02\end{array}$ \\
\hline \multicolumn{9}{|c|}{ South West Thames region } \\
\hline $\begin{array}{l}\text { Wandsworth } \\
\text { West Surrey and NE Hampshire } \\
\text { Chichester } \\
\text { Worthing }\end{array}$ & $\begin{array}{l}187676 \\
277738 \\
183196 \\
243777\end{array}$ & $\begin{array}{l}176362 \\
283592 \\
201127 \\
258619\end{array}$ & $\begin{array}{l}159427 \\
261135 \\
238675 \\
335366\end{array}$ & $\begin{array}{l}165155 \\
248533 \\
230647 \\
315988\end{array}$ & $\begin{array}{r}-7 \cdot 87 \\
0 \cdot 11 \\
7 \cdot 64 \\
4 \cdot 01\end{array}$ & $\begin{array}{r}-9 \cdot 60 \\
-7 \cdot 92 \\
18 \cdot 67 \\
29 \cdot 68\end{array}$ & $\begin{array}{r}3 \cdot 59 \\
-4 \cdot 83 \\
-3 \cdot 36 \\
-5 \cdot 78\end{array}$ & $\begin{array}{r}-13 \cdot 72 \\
-12 \cdot 27 \\
23 \cdot 44 \\
27 \cdot 09\end{array}$ \\
\hline \multicolumn{9}{|c|}{ Wessex region } \\
\hline $\begin{array}{l}\text { Basingstoke and NE Hampshire } \\
\text { Winchester } \\
\text { East Dorset } \\
\text { Isle of Wight }\end{array}$ & $\begin{array}{l}225412 \\
214718 \\
453297 \\
129409\end{array}$ & $\begin{array}{l}242877 \\
221663 \\
484441 \\
136212\end{array}$ & $\begin{array}{l}223615 \\
217892 \\
577088 \\
158900\end{array}$ & $\begin{array}{l}210932 \\
211465 \\
535082 \\
153473\end{array}$ & $\begin{array}{l}5 \cdot 64 \\
1 \cdot 22 \\
4 \cdot 78 \\
3 \cdot 20\end{array}$ & $\begin{array}{r}-7 \cdot 93 \\
-1 \cdot 70 \\
19 \cdot 12 \\
16 \cdot 66\end{array}$ & $\begin{array}{l}-5 \cdot 67 \\
-2 \cdot 95 \\
-7 \cdot 28 \\
-3 \cdot 42\end{array}$ & $\begin{array}{l}-8 \cdot 25 \\
-3 \cdot 44 \\
15 \cdot 73 \\
16 \cdot 28\end{array}$ \\
\hline \multicolumn{9}{|c|}{ Oxford region } \\
\hline $\begin{array}{l}\text { Wycombe } \\
\text { Oxfordshire } \\
\text { Kettering } \\
\text { Milton Keynes }\end{array}$ & $\begin{array}{l}271482 \\
558276 \\
264187 \\
187869\end{array}$ & $\begin{array}{l}251872 \\
580406 \\
275356 \\
251052\end{array}$ & $\begin{array}{l}254921 \\
545913 \\
273107 \\
201805\end{array}$ & $\begin{array}{l}238286 \\
509411 \\
272147 \\
202310\end{array}$ & $\begin{array}{r}-9.04 \\
1.93 \\
2.19 \\
31.02\end{array}$ & $\begin{array}{r}1.21 \\
-5.94 \\
-0.82 \\
-19.62\end{array}$ & $\begin{array}{r}-6.53 \\
-6.69 \\
-0.35 \\
0.25\end{array}$ & $\begin{array}{r}-13.94 \\
-10.54 \\
1.00 \\
5.58\end{array}$ \\
\hline \multicolumn{9}{|c|}{ South Western region } \\
\hline $\begin{array}{l}\text { Southmead } \\
\text { Frenchay } \\
\text { Exeter } \\
\text { Torbay }\end{array}$ & $\begin{array}{l}236601 \\
220386 \\
316608 \\
248189\end{array}$ & $\begin{array}{l}238187 \\
228024 \\
331264 \\
278813\end{array}$ & $\begin{array}{l}234897 \\
235675 \\
386689 \\
330643\end{array}$ & $\begin{array}{l}231578 \\
221179 \\
365167 \\
312416\end{array}$ & $\begin{array}{r}-1 \cdot 30 \\
1 \cdot 44 \\
2 \cdot 58 \\
10 \cdot 14\end{array}$ & $\begin{array}{r}-1 \cdot 38 \\
3 \cdot 36 \\
16 \cdot 73 \\
18 \cdot 59\end{array}$ & $\begin{array}{l}-1 \cdot 41 \\
-6 \cdot 15 \\
-5 \cdot 57 \\
-5 \cdot 51\end{array}$ & $\begin{array}{r}-4 \cdot 04 \\
-1 \cdot 60 \\
13 \cdot 08 \\
23 \cdot 42\end{array}$ \\
\hline \multicolumn{9}{|c|}{ West Midlands region } \\
\hline $\begin{array}{l}\text { West Birmingham } \\
\text { Solihull } \\
\text { North Birmingham } \\
\text { Herefordshire }\end{array}$ & $\begin{array}{l}209069 \\
203474 \\
160550 \\
158880\end{array}$ & $\begin{array}{l}196142 \\
207421 \\
168709 \\
169373\end{array}$ & $\begin{array}{l}181155 \\
207322 \\
164221 \\
178129\end{array}$ & $\begin{array}{l}198644 \\
193571 \\
172722 \\
173884\end{array}$ & $\begin{array}{r}-8.02 \\
-0.05 \\
3.03 \\
4.52\end{array}$ & $\begin{array}{r}-7 \cdot 64 \\
-0.05 \\
-2 \cdot 66 \\
5 \cdot 17\end{array}$ & $\begin{array}{r}9 \cdot 65 \\
-6 \cdot 63 \\
5 \cdot 18 \\
-2 \cdot 38\end{array}$ & $\begin{array}{r}-6 \cdot 84 \\
-6 \cdot 73 \\
5 \cdot 48 \\
7 \cdot 30\end{array}$ \\
\hline \multicolumn{9}{|c|}{ Mersey region } \\
\hline $\begin{array}{l}\text { St Helens and Knowsley } \\
\text { South Sefton } \\
\text { Wirral } \\
\text { Southport and Formby }\end{array}$ & $\begin{array}{l}346061 \\
177033 \\
353733 \\
122216\end{array}$ & $\begin{array}{l}335110 \\
165986 \\
356362 \\
130110\end{array}$ & $\begin{array}{l}320187 \\
168213 \\
364613 \\
137905\end{array}$ & $\begin{array}{l}336914 \\
172540 \\
382792 \\
144781\end{array}$ & $\begin{array}{r}-5 \cdot 06 \\
-8 \cdot 07 \\
-1 \cdot 23 \\
4 \cdot 38\end{array}$ & $\begin{array}{r}-4 \cdot 45 \\
1 \cdot 34 \\
2 \cdot 32 \\
5 \cdot 99\end{array}$ & $\begin{array}{l}5 \cdot 22 \\
2 \cdot 57 \\
4 \cdot 99 \\
4 \cdot 99\end{array}$ & $\begin{array}{r}-4 \cdot 55 \\
-4 \cdot 44 \\
6 \cdot 10 \\
16 \cdot 15\end{array}$ \\
\hline \multicolumn{9}{|c|}{ North Western region } \\
\hline $\begin{array}{l}\text { Central Manchester } \\
\text { North Manchester } \\
\text { Lancaster } \\
\text { Blackpool, Wyre, and Fylde }\end{array}$ & $\begin{array}{l}116886 \\
143106 \\
134119 \\
320849\end{array}$ & $\begin{array}{l}105873 \\
121958 \\
137207 \\
332466\end{array}$ & $\begin{array}{l}101847 \\
121060 \\
149141 \\
376411\end{array}$ & $\begin{array}{l}107603 \\
138168 \\
149141 \\
391932\end{array}$ & $\begin{array}{r}-11 \cdot 19 \\
-16 \cdot 44 \\
0.30 \\
1.59\end{array}$ & $\begin{array}{r}-3 \cdot 80 \\
-0 \cdot 74 \\
8 \cdot 70 \\
13 \cdot 22\end{array}$ & $\begin{array}{r}5 \cdot 65 \\
14 \cdot 13 \\
0 \cdot 00 \\
4 \cdot 12\end{array}$ & $\begin{array}{r}-9 \cdot 74 \\
-5 \cdot 34 \\
9 \cdot 03 \\
19 \cdot 77\end{array}$ \\
\hline
\end{tabular}


THE OVERALL GAINERS

Overall, however, although the seaside resorts tended to lose on the mortality weighting, the loss was much less than the gains due to the age weights, so that they emerged as the major gainers.

\section{Discussion}

Disaggregating the components in the national capitation formula shows complex interactions between the three main elements, leading to much larger swings at district than at regional level. The range of these swings was $-17 \%$ to $28 \%$ at district level but only $-9 \%$ to $6 \%$ at regional level. Regions which included inner city and seaside resorts tended to have the widest range of gainers and losers.

The combination of relatively large gainers and losers within certain regions seems likely to pose problems with pace of adjustment in these regions The magnitude of the changes suggests that some degree of discretion will continue to be required in the capitation funding of districts

\section{AGE-WEIGHTS}

Since age weights accounted for the bulk of the total gains in those relatively few districts with large gains, the robustness of the estimation of these weights deserves particular attention. As the age weights imply that about $20 \%$ of total capitation funding goes to the over $85 \mathrm{~s}$, with over $50 \%$ of total funding to the over $65 \mathrm{~s}$, the importance of the elderly is clear.

Although these age weights have been routinely reported in Department of Health publications, ${ }^{6}$ the calculations on which they are based have received little attention. ' Apart from problems about the degree to which they are based on the appropriate units (for example, admissions or bed days) and the quality of the data (particularly in relation to unit costs), the interface between the NHS and other services also requires attention. In particular, patterns of service use among the elderly are likely to be affected by the availability of nursing and residential home places.

\section{POPULATION CHANGES}

Furthermore, the impact of selective migration by the elderly on population health requirements in both home and host districts remains unclear. The use of the under 75 all cause standardised mortality ratio could mitigate such effects, but to an unknown degree. In general, much more remains to be known about the actual and the most appropriate patterns of service use by elderly people not only of NHS services but also of services provided by local authorities, voluntary agencies, and the private sector. The scope for discretion by regions to allow for such variations without appearing to be biased remains to be determined.

Population projections also played an important part in the total projected changes. What matters is not only the total projected population of each district but also that of each of the age groups. The highest age-cost weights apply to two relatively small groups, births and the over $85 \mathrm{~s}$, each of which amount to about 750000 people nationally, or 3500 in each district. Greater imprecision inevitably surrounds projections for such relatively small groups than for the total population, an imprecision which is then magnified by the high age weights attached to these groups.

\section{ADJUSTING FOR MORTALITY}

Despite the intense debate over the use of a mortality adjustment in the capitation formula, it had much less impact than either the population projections or the age weights. The use of the square root of the standardised mortality ratio rather than the full standardised mortality ratio has the effect of attenuating the mortality weight by around half. The case for the use of the square root of mortality, which was based on large scale analysis of hospitalisation rates by electoral wards in six regions, ${ }^{15}{ }^{16}$ has, however, been subject to considerable criticism. ${ }^{17}$

\section{Conclusions}

The robustness of the methods used to derive projected capitation populations requires re-examination to ensure that changes of the magnitude shown here are well founded. The intensity of the debate over the adjustments for mortality and social deprivation may well reflect unease the scale of change, although it is the application of age-cost weights to projected populations that accounts for the bulk of the change in those districts projected to gain or lose the most. The worry must be that unless the age-cost weights are well founded their application could lead to much larger swings in funding than those associated with the workings of the internal market. Such large changes in funding could have irreversible effects, such as the closure of units.

More research is required on the health needs and associated costs of the elderly, particularly the over $85 \mathrm{~s}$, including patterns of selective migration and interfaces with other types of care such as nursing and residential homes.

Finally, since a level of uncertainly surrounds estimation of each of the elements of the capitation formula, the formula should be used only with caution and over the shorter term. Although the use of five year standardised mortality ratios and three year age weights reduce some of the uncertainty, the inevitable lack of precision in projections of relatively small age groups suggests the need for close monitoring of population changes year by year. There may well be scope for incorporating such uncertainties into the capitation formula, perhaps along the lines recently suggested for dealing with similar problems in funding local authorities. ${ }^{20}$

1 Mays N, Bevan G. Resource allocation in the health service: a review of the resource allocation working party. London: Bedford Press, 1987.

2 Resource Allocation Working Party. Repon. London: DHSS, 1976.

2 Resource Allocation Working Party. Report. London: DHSS, 1976.

3 Winyard PGA. RAWP-new injustice for old? BMF 1981;286:1112.
4 Funding and contracts for hospital services. London: HMSO, 1989. (Working for patients working paper 2 .)

5 Sheldon TA, Davey-Smith G, Bevan G. Weighting in the dark: resource allocation in the new NHS. BMF 1993;306:835-9.

6 Department of Health and Office of Population Censuses and Surveys. Report. London: HMSO, 1993.

7 NHS Management Executive. Hospital and community health services 1993-4. Cash limits exposition booklet. Leeds: NHSME, 1993.

8 Review of RAWP. London: DHSS, 1987

9 Senn SJ, Shaw H. Resource allocation: some problems in applying the national formula to area and district revenue allocations. I Epidemiol Comm Health 1978;22:32-7.

10 Snaith AH. Subregional resource allocations in the National Health Service. 7 Epidemiol Community Health 1978;22:16-21.

11 Geary K. Technical deficiencies in RAWP. BMf 1977; ; 1367

12 Palmer S, West P, Dodd P. Randomness in the RAWP formula: the reliability of mortality data in the allocation of National Health Service revenue. of mortality data in the allocation of

13 Pinder DC. Catchment populations: the properties and accuracy of various methods for their estimation. Community Medicine 1982;4:188-95.

14 NHS Management Executive. Public health common dataset. Leeds: NHSME, 1992.

15 Coopers and Lybrand. Integrated analysis for the review of RAWP. London: Coopers and Lybrand, 1988

16 Royston GHD, Hurst JW, Lister EG, Sewart PA. Modelling the use of health services by populations of small areas to inform the allocation of centra resources to larger regions. Socio-economic Planning 1992;26:168-80.

17 Carr-Hill R, Maynard AK, Slack RT-MR. Morbidity variation and RAWP. f Epidemiol Comm Health 1990;44:271-3.

18 Sheldon TA, Carr-Hill R. Resource allocation by regression on the Nationa Health Service; a critique of the Resource Allocation Working Party's Review. Fournal of the Royal Statistical Society Association 1992;155:403-20.

19 Copas JB. A formula for the allocation of resources based on uncertain prediction of need. Foumal of the Royal Statistical Society Association 1993;156:107-13.

(Accepted 12 August 1993) 